\title{
Conservative treatment for well-differentiated endometrial cancer: when and why it should be considered in young women
}

\author{
Michele Peiretti ${ }^{1}$, Francesca Congiu ${ }^{1}$, Enzo Ricciardi ${ }^{2}$, Paolo Maniglio $^{3}$, Valerio Mais ${ }^{1}$ and Stefano Angioni ${ }^{1}$ \\ ${ }^{1}$ Department of Surgical Sciences, Division of Gynecology and Obstetrics, University of Cagliari, 09124 Cagliari, Italy \\ ${ }^{2}$ Department of Gynäkologie and Gynäkologische Onkologie, Kliniken Essen-Mitte, 45136 Essen, Germany \\ ${ }^{3}$ Department of Scienze Medico-Chirurgiche e di Medicina Traslazionale, Sapienza University of Rome, 00185 Rome, Italy
}

Correspondence to: M Peiretti. Email: michelepeiretti@hotmail.com

\begin{abstract}
The aim of this review was to update current knowledge on the conservative treatment of endometrial cancer (EC) based on a literature review. A web-based search in the MEDLINE database was carried out on EC management and treatment. All relevant information has been collected and analysed. Case series were mainly found in the literature search. Conservative treatments were offered to young patients with stage I low-grade endometrioid carcinomas of the endometrium. Different options included high/low dose progestin treatments, hysteroscopic resection of the disease, a levonorgestrel intrauterine device or a combination of various strategies. The overall complete response rate was near $76.5 \%$ with a recurrence rate of up to $33.8 \%$. Pregnancy outcomes reached rates of $64.8 \%$ for live births. The current clinical outcomes show that conservative treatment aimed at preserving fertility is feasible for stage I endometrial well-differentiated adenocarcinomas in motivated patients under close monitoring.
\end{abstract}

Keywords: endometrial cancer, fertility-sparing treatment, hysteroscopic resection, conservative treatment

ecancer 2019, 13:892 https://doi.org/10.3332/ecancer.2019.892

Copyright: $\odot$ the authors; licensee ecancermedicalscience. This is an Open Access article distributed under the terms of the Creative Commons Attribution License (http://creativecommons.org/licenses/by/3.0), which permits unrestricted use, distribution, and reproduction in any medium, provided the original work is properly cited. 


\section{Introduction}

Endometrial cancer (EC) is the most common gynaecologic malignancy in the United States. It accounts for $3.6 \%$ of all cancers (incidence rate of 26.1/100,000 women) and it generally affects postmenopausal women, with a median age at diagnosis of 62 years [1]. EC tends to be diagnosed at an early stage in most women and is usually associated with a good prognosis. Transvaginal ultrasound (US) and diagnostic hysteroscopy are the main diagnostic procedures in the diagnosis. Hysteroscopy provides direct visualisation of the endometrial cavity, thereby allowing a targeted biopsy or excision of the lesions identified during the procedure [2]. Miniaturised devices and the development of an office approach allow an accurate and reliable diagnosis in the majority of women with abnormal uterine bleeding or with abnormal uterine findings at USs [3]. Abnormal uterine bleeding is the most common symptom and a preoperative diagnosis should be mandatory to avoid unexpected malignancy and consequently, inappropriate surgical treatment and possible spreading of the disease [4]. Standard treatment of early-stage EC includes hysterectomy without vaginal cuff, bilateral salpingo-oophorectomy with sentinel lymph node mapping or systematic pelvic and para-aortic lymph node dissection [10, 11]. In those patients older than 45 years with G2-G3 and myometrial invasion, bilateral salpingo-oophorectomy is recommended to avoid the risk of stimulating an undetected metastatic disease [4].

Young patients generally have a history of oligomenorrhoea, chronic anovulation and infertility, obesity, polycystic ovary syndrome (PCOS) and diseases related to the exposure of oestrogens and androstenedione [5-7]. Moreover, they tend to have a more favourable prognosis considering the frequent low grading and no evidence of myometrial invasion [6, 8, 9]. In fact, when the disease is well differentiated and confined to the uterus, the 5 -year survival rate is $96 \%$ [1].

The endometrioid type is the most prevalent, accounting for $90 \%$ of all cases. High-grade tumours, such as the clear cell and undifferentiated types, are present in the remaining cases and these are considered characteristic of patients of an advanced age [4].

Young patients who are willing to preserve fertility may prefer a conservative treatment and should be carefully evaluated with appropriate consultation. A myometrial invasion and the grading of the neoplasm should be identified, these being the main prognostic factors and indications for a possible conservative treatment [4]. Despite the fact that these two parameters are good indicators of the spread of the disease, patients with grade 1 disease have about $3 \%$ and $2 \%$ of pelvic and para-aortic dissemination, respectively [13]. In cases of grade 1 lesions that are confined to the endometrium, the rate of nodal metastasis is negligible. Therefore, the exclusion of any myometrial involvement in grade 1 lesions should be regarded as an indication for conservative treatment [14].

Several studies have tried to evaluate a more specific and sensitive test in evaluating the involvement of the myometrium. Hardesty et al [15] reported a comparison between magnetic resonance imaging (MRI) and spiral computed tomography (CT) scans, showing that MRI is more sensitive and specific in the detection of myometrial invasion and cervical involvement. Comparisons between MRI and transvaginal US showed that both these techniques produce similar results [16, 17]. Saez et al [18] reported a higher diagnostic accuracy of the MRI with gadolinium. This same group also confirmed findings that emphasise the better diagnostic results obtained through the MRI with contrast agents [19]. Literature data show MRI sensitivity and specificity of $83 \%$ and $82 \%$, respectively, so it can be considered an effective but still not a perfect diagnostic tool [20].

Therefore, all patients opting for a conservative treatment have to be adequately informed of the possibility of false negatives and their relative consequences. For those patients who are candidates for conservative treatment, a laparoscopy with peritoneal cytology, lymph node biopsy and an evaluation of the adnexa should be offered [12].

The following criteria must be met for appropriate screening of young patients wanting to preserve fertility:

(a) Well-differentiated carcinoma

(b) Myometrial invasions absent on the MRI, CT scan and pelvic US (trans-abdominal-trans-vaginal)

(c) No pelvic and para-aortic lymph node involvement

(d) No ovarian cancer

(e) No contraindications to medical treatments

(f) Patients who strongly wish to preserve fertility

In addition, the patient has to agree to attend a precise follow-up after the treatment. 
The aim of the present review consists of an evaluation of the various medical strategies for well-differentiated early-stage EC that a gynaecologic oncologist should offer to these women. We particularly focused on contemporary clinical trials and the evidence surrounding conservative management.

\section{Materials and methods}

An electronic literature search via the MEDLINE database was performed to identify published English-language articles addressing the conservative treatment of women with a histologic diagnosis of grade 1 adenocarcinoma of the endometrium.

We selected reports published between January 2004 and December 2017 using the PubMed database. Keywords and terms searched included: conservative treatment, endometrial cancer, progestin therapy and fertility. The search was further narrowed by limiting articles cited to only those reporting on humans.

We excluded studies that described hormonal therapy in the following settings: high-risk (grade 2 or 3 ) endometrial adenocarcinoma, advanced-stage EC and non-endometrioid type. We also excluded case reports or papers in which EC data were not clearly distinguished from atypical hyperplasia (AH) ones or in which considered patients included also postmenopausal women. Furthermore, papers with incomplete results on oncological or pregnancy outcomes were excluded. The results of selected papers were carefully evaluated to establish their relevance. (Figure 1).

From each relevant study, we analysed demographic data: patient's age, body mass index (BMI), parity, comorbidities, histology, different types of hormonal agents, routes of administration and method and timing of intervals for endometrial re-evaluations. Information regarding oncologic and fertility outcomes was recorded, including response rates. A response of the grade 1 adenocarcinoma of the endometrium was defined as 'complete regression' when the final specimen showed an atrophic, proliferative or secretory endometrium. When no change was detected in histological specimens, we defined it 'stable disease', when pathology showed regression of lesions from Cancer to Hyperplasia, a 'partial remission' was defined. 'Progressive disease' was diagnosed when follow-up tests demonstrated a higher grade of disease or myometrial invasion, while 'Relapse' was defined as positivity of pathological analysis after one or more negative specimens.

Data regarding reproductive outcomes were also collected, including the number of patients who became pregnant and the number of live births, both from patients who conceived spontaneously and with assisted reproductive technologies (ART); however, this information was not reported in all studies.

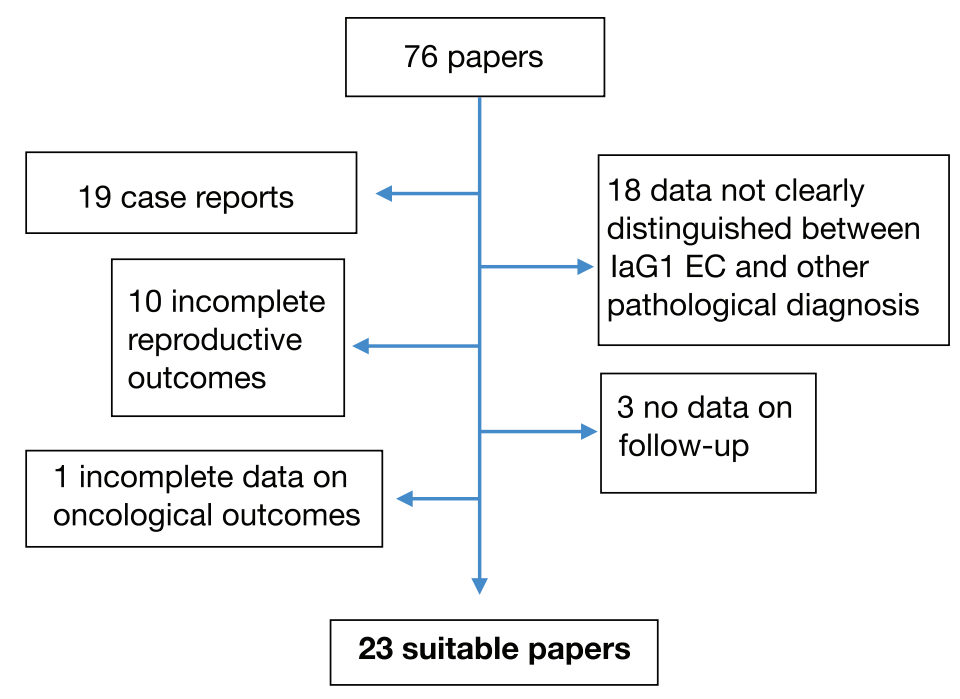

Figure 1. Selection of suitable papers for our review. 
We analysed results from prospective/retrospective studies published to date, focusing on all controversies surrounding fertility-sparing treatment for well-differentiated EC.

\section{Results}

We identified 23 studies, including prospective/retrospective series. From these studies, we identified 299 patients with a diagnosis of welldifferentiated endometrial adenocarcinoma who were treated with various hormonal scheduled therapies, combined, in some studies, with hysteroscopic resection of the tumour.

The median age for the overall cohort was 31, 45 years (range: 18-47 years). Median BMI was 24 (range 17.4-77.6). Data regarding parity were available only for 184 patients. Of these, 148 patients $(79 \%)$ were nulliparous. Seventeen studies reported associated conditions, as PCOS, menstrual irregularities, diabetes mellitus, hypertension, familiar history positive for Lynch syndrome or other tumours. (Demographic data and treatment features are showed in Table 1.)

Hysteroscopic resection of neoplastic lesions preceded medical treatment in four studies (76 patients, $25 \%$ of total).

The most commonly used drugs were megestrol acetate, medroxyprogesterone acetate (MPA), levonorgestrel (LNG) intrauterine device (IUD), aromatase inhibitors (letrozole, anastrozole), analogue of gonadotropin-releasing hormone (GnRH) (as leuprolide), combined oral contraceptives, lynestrenol, tamoxifen, norethisterone acetate and hydroxyprogesterone caproate. In most studies, more than one drug was used, so it was not possible to specify how many patients took every drug.

After a median follow-up of 39 months (range: $1.5-412$ months), the complete regression rate was $76.5 \%$ (228 patients) with a relapse rate of $33.5 \%$ (77 patients) between patients who had a complete response (CR).

Table 1. Demographic data and treatment features.

\begin{tabular}{|l|l|l|}
\hline \multicolumn{3}{|c|}{ Demographic data $(n=299)$} \\
\hline Median age & 31,45 years & $18-47$ years \\
\hline Median BMI & 24 & $17.4-77.6$ \\
\hline Nulliparous (data available only for 184 patients) & 148 & $79 \%$ \\
\hline Hysteroscopic resection prior to medical treatment & 4 studies, 76 patients & $25 \%$ \\
\hline Pharmacological agents & & \\
I. $\quad$ Megestrol Acetate & 18 studies & \\
II. MPA & 14 studies & \\
III. LNG-IUD & 5 studies & \\
IV. Letrozole/Anastrozole & 1 study & \\
V. GnRH-a (Leuprolide) & 2 studies & \\
VI. Combined Oral Contraceptives & 1 study & \\
VII. Lynestrenol & 1 study & \\
VIII. Tamoxifen (always in association with other drugs) & 2 studies & \\
IX. Norethisterone acetate & 2 studies & $2-49$ months \\
X. Hydroxyprogesterone caproate & 1 study & \\
\hline Median duration of therapy & 6 months & \\
\hline
\end{tabular}


Table 2. Oncological outcomes.

\begin{tabular}{|l|c|c|}
\hline \multicolumn{3}{|c|}{ Oncological outcomes $(n=299)$} \\
\hline CR & 228 & $76.25 \%$ of total \\
\hline NR & 65 & $21.7 \%$ of total \\
\hline SD & 33 & $50.8 \%$ of NR \\
\hline PD & 32 & $49.2 \%$ of NR \\
\hline PR & 7 & $2.3 \%$ of total \\
\hline Relapse & 77 & $33.8 \%$ of CR \\
\hline
\end{tabular}

Oncological outcomes are shown in Table 2. Sixty-five patients (21.7\%) did not respond to therapy. Of these patients, 33 (50.8\%) showed stable disease (SD) and $32(49.2 \%)$ showed progressive disease (PD). Seven patients (2.3\%) experienced partial remission (PR).

Follow up consisted in dilatation and curettage (D\&C) (69.6\% of studies) or any other endometrial sampling (as hysteroscopic biopsy or Pipelle) in $100 \%$ of studies, in 11 studies in transvaginal US ( $47.8 \%$ of studies), MRI ( $26 \%$ of studies), at a median interval between evaluations of 3 months.

Data regarding surgical management are the following: 136 women (45.6\%) had a hysterectomy. Only four studies reported the interval of time between diagnosis and hysterectomy. This interval ranged from 6 to 84 months, with a median of 12 months. Finally, data on histology were reported for 132 women. Of these, 33 had negativity for any malignancy, 59 revealed IAG1 EC, 9 showed PR to AH and 22 showed progression (because of increased grading or staging). In particular, nine women had simultaneous ovarian implants of endometrioid adenocarcinoma.

Data regarding adverse effects were collected. Only six studies reported adverse effects, and six specified the absence of any side effect. Among the studies considered, five reported weight gain, three reversible liver dysfunction, one swelling and cutaneous hyperpigmentation and one study reported hot flushes and vaginal dryness (this study contemplated the use of GnRH analogues).

Regarding reproductive outcome, data are shown in Figure 2. Studies reported a total of 119 pregnancies (among 162 women who attempted to conceive, pregnancy rate $73.4 \%$ ), which included women who conceived both spontaneously (54 pregnancies) and via ART (65). In particular, 97 women referred to the ART centre. Of the 119 analysed pregnancies, 105 live births occurred (54 from ART).

\section{Discussion}

Given the hormonal dependency of EC, several groups have mainly adopted medical treatments with high doses of progestins, while others utilised gonadotropin-releasing hormone agonists $(\mathrm{GnRHa})$ or antiandrogens [21-23]. It has been well established that progestins, besides many other systemic effects, may cause endometrial atrophy and are widely used in the treatment of many gynaecological benign conditions [24]. Considering the hormonal dependency of the majority of ECs, hormonal therapy could lead to a tumoural quiescence or even a disappearance with treatment [25]. Specific mechanisms explaining how progestins acting as oestrogen antagonists can cause their effect on endometrial carcinomas could be related to apoptosis pathways [25] and more the recently described Phospatase and Tensin Homolog gene mutation pathways [24].

Progestins have been utilised for years in the treatment of metastatic EC because they act as oestrogen antagonists and inhibit the mitosis of endometrial cells and favour apoptosis [26]. Some of these also have anti-angiogenic effects [27-29]. Several groups, as described below, have utilised MPA 100-800 mg one time per day (QD) for 4-14 months. 


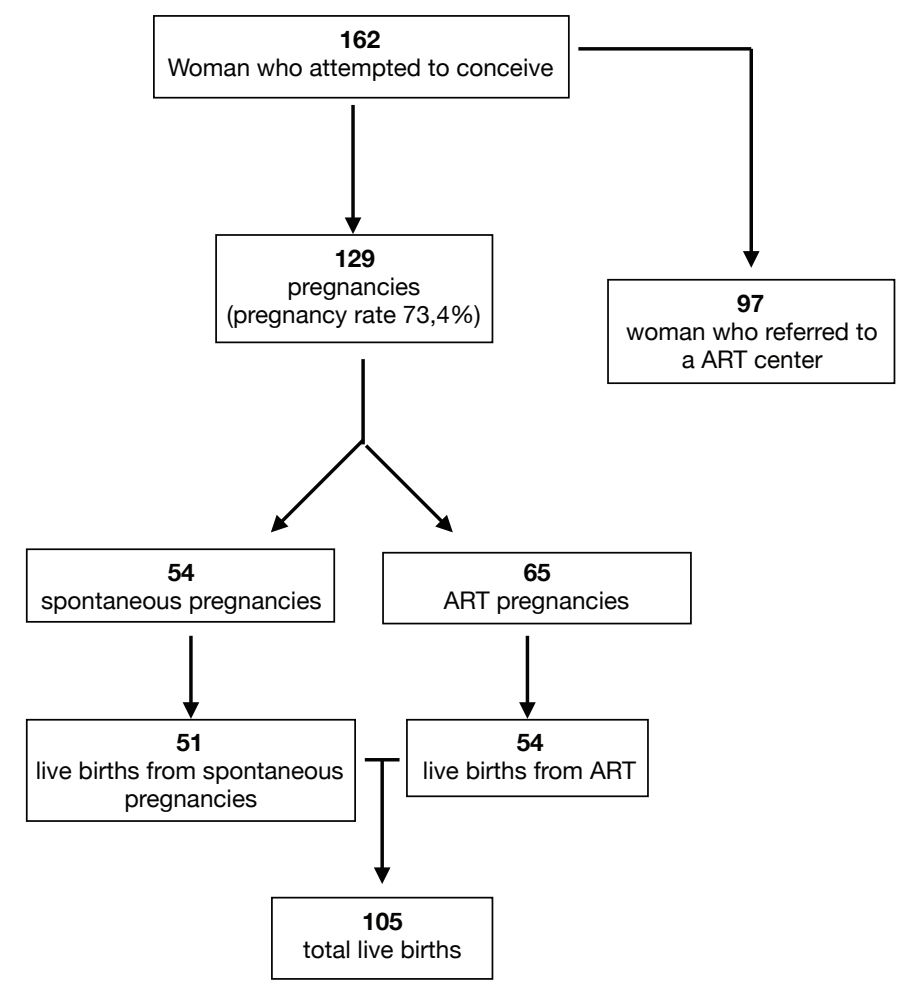

Figure 2. Reproductive outcomes.

Kaku et al [21] reported treating 12 patients with EC with MPA 200-400 mg QD. Of the 12 patients, eight had a CR to treatment and one patient had a partial response. The average length of treatment was 3 months. The average follow-up examination occurred at 24 months for responding patients and showed a case of recurrence treated with a second cycle of MPA that responded to therapy. Ferrandina et al [30] reported on a 30-year-old patient who was nulliparous with a well-differentiated endometrial adenocarcinoma. This patient was treated from the 15th to the 25th day following the onset of menstruation with dihydrogesterone 20 mg QD. The patient subsequently responded to medical treatment and had a successful pregnancy and delivery. Eight months later, regular follow-up MRI and CT scan showed distant metastases and an exploratory laparotomy revealed parametrial, ovarian, cervical and omental metastases. Kimming et al [31] reported a case of a 28-year-old patient who was nulliparous with a well-differentiated adenocarcinoma treated with MPA 200 mg QD for 8 weeks. After medical treatment, the diagnostic hysteroscopy and biopsy revealed pathology regression. The patient then underwent gamete intrafallopian transfer and successfully carried triplets to term and delivered three live foetuses. A year after delivery, the patient did not show any signs of recurrent disease or distant repetitions. Randall and Kurman reported on a study conducted on 12 women with well-differentiated adenocarcinomas who were younger than 40 years of age and treated with high doses of progestins. Nine patients had a CR to treatment after 9 months, however, three patients still had the disease. None of the responding patients showed signs of recurrent or progressing disease after an average follow-up of 40 months [32]. Yamazawa et al [8] reported on nine women averaging 36 years of age with endometrial adenocarcinomas and treated with MPA $400 \mathrm{mg}$ QD for 6 months. Of the nine patients, 78\% had a CR and 22\% a partial one. The overall rate of response was $100 \%$ after 6 months of treatment with MPA. Ramirez et al [33] published a review comprised of 27 publications on the conservative treatment of 81 patients. Half of the patients were treated with MPA 200-600 mg QD. Although approximately $75 \%$ of the patients had a CR to treatment within an average of 12 weeks, the average length of treatment was 6 months. Furthermore, $76 \%$ had a recurrence after an average of 20 months and $30 \%$ of these patients with recurrence underwent a second cycle of progestins with a response in $80 \%$ of the cases. We may conclude that for half of the cases, fertility was preserved, thanks to hormonal therapy with high doses of progestin, and that $25 \%$ of the cases that failed to respond to the first therapeutic cycle had complete recovery after the second cycle. 
After a detailed analysis of the previous clinical experiences, there is no definitive consensus regarding the optimal progestin regimen and timing, and most studies assessed in this review did not report long follow-up times following hormonal treatments. Progestin therapy has an impact on the endometrial cells as early as 10 weeks after the initiation of treatment. However, most recognise the need for a minimum of 3 months of treatment before assessing a response with endometrial hyperplasia and even longer for EC [34-36]. Thus, some clinicians use this time frame in determining responsiveness to progestin therapy.

An innovative approach to the conservative treatment of endometrial carcinomas requires hysteroscopic resection of EC along with part of the adjacent myometrium and subsequent medical therapy with progestin. Two Italian series described a total of 20 patients treated with a similar hysteroscopic technique in which the authors observed only one recurrence after a median follow-up of 40 and 50.5 months in five patients with six pregnancies. This technique may be considered for those cases with small lesions even though there are possible complications, such as spreading of the disease, intrauterine adhesions and obstetric complications due to myometrial damage [37-39]. In particular, placenta accreta is the most dangerous eventuality in these patients because of the altered vascularity of damaged tissues and higher invasivity of trophoblast in scars [40]. However, it should be considered in selected cases. A recent review of the literature reported a recurrence rate of $11 \%$ after a median follow-up period of 40 months (range: 11-82 months) after hysteroscopic resections of endometrial lesions and subsequent hormonal therapy with oral and/or IUD progestins [41].

Another interesting therapeutic option is the use of the levonorgestrel-releasing-IUD (LNG-IUD) plus GnRH. Some case reports have been published with a 100\% CR rate [42, 43]. Minig L et al [44] treated 34 patients with a combination of LNG-IUD for 1 year followed by 6 months of $\mathrm{GnRH}$ treatment in patients younger than 40 years of age. The $\mathrm{CR}$ rate was $95 \%$, although the relapse rate among patients with atypical endometrial hyperplasia was $5 \%$ compared to $28 \%$ among patients diagnosed with EC.

To find the best evidence, we need to wait for the results of the following two trials (both recruiting patients): phase II trial from MD Anderson (registry \# NCT0078861) on the use of LNG-IUD for the treatment of endometrial hyperplasia and low-grade endometrial carcinomas, and the feMMe trial, by Obermair et al [45] (registry \# NCT01686126), which aims to study the efficacy and safety of LNG-IUD + Metformin + weight loss in patients at an early stage of EC. These studies could clarify a specific role for the use of LNG-IUD in this disease.

Data selected for our review show a significant response rate for EC patients treated conservatively ( $76.25 \%$ achieved CR), but there is a significant relapse rate- $33.8 \%$ of patients who had a complete remission). Thus, it seems fundamental to adequately select patients and carefully counsel them before starting a conservative approach. It is also necessary to plan a comprehensive follow-up to diagnose eventual disease stability ( $50.8 \%$ of non-responders (NR) in our review), progressions $(49.2 \%$ of NR) or relapses and propose the patient for further medical therapy or standard surgery.

After a complete pathological response, all patients should attempt pregnancy. In those women who do not become pregnant after 3 months, it is advised to carry out first-stage examinations for the evaluation of an infertile couple. If the woman has a history of infertility or chronic anovulation and has no signs of disease, ovulation induction may be considered. Despite the exposure to high levels of oestrogens, a higher risk of recurrence has not been shown; in fact, the utilisation of MPA may reduce the waiting time of those wanting a pregnancy [46-49].

From our international literature review, we found a wide range of pregnancy successes (overall pregnancy rate $73.4 \%$ ) among young women with EC after conservative treatment. The tumours were well differentiated in all of the selected studies, and the pregnancies were obtained through ART in $51.4 \%$ of the cases.

All patients should consider demolitive surgery as soon as possible after achieving pregnancy or after the failure of several ART procedures.

In our set of data, hysterectomy with pathological evaluation was reported for $132(44 \%)$ patients. The surgical procedure was performed after a medium period of 30 months after diagnosis, reflecting the interval necessary for therapy and ART to be accomplished. Between all the patients who underwent surgical procedure, only 33 patients $(25 \%)$ showed no evidence of disease at pathological evaluation, while $22(16.7 \%)$ showed a progression of the disease, thus pointing out the importance of surgery in all patients diagnosed with EC, even if conservative therapy was effective in a short period.

There are some limitations to this review. First, the main source was a group of retrospective studies, there may have been inherent reporting and observational biases and a lack of long-term follow-up data for many studies. Additionally, details of treatments and surgical pathologies were often not available and there was no centralised pathological review.

Some included studies were not of high quality, so we conclude that large prospective or randomised studies comparing the efficacy of hormonal agents and associated reproductive outcomes are warranted. 


\section{Conclusion}

Conservative treatment with medical therapy with or without hysteroscopic resection of the lesion should be considered in selected young patients with grade 1 stage I EC who wish to preserve fertility. These patients should undergo comprehensive follow-up after achievement of $\mathrm{CR}$. This is important because, despite high CR rates, it seems fundamental to identify patients who did not respond, who had PD or who relapsed.

Patients should also attempt pregnancy as soon as possible after the CR, spontaneously or via ART, to minimise the risk of relapse.

After the achievement of pregnancy, or after the failure of ART, standard surgery should be offered to patients.

\section{Funding and conflicts of interest}

The authors of the present publication did not receive any research support funding and declare no conflict of interest.

\section{References}

1. National Cancer Institute Cancer stat facts: uterine cancer [https://seer.cancer.gov/statfacts/html/corp.html] Date accessed: $5 / 3 / 18$

2. Angioni S, Loddo A, and Milano F, et al (2008) Detection of benign intracavitary lesions in postmenopausal women with abnormal uterine bleeding: a prospective comparative study on outpatient hysteroscopy and blind biopsy J Minim Invasive Gynecol 15(1) 87-91 https://doi.org/10.1016/j.jmig.2007.10.014 PMID: 18262151

3. Di Spiezio Sardo A, Bettocchi S, and Spinelli M, et al (2010) Review of new office-base hysteroscopic procedure 2003-2009 J Minim Invasive Gynecol 17(4) 436-448 https://doi.org/10.1016/j.jmig.2010.03.014 PMID: 20621007

4. Colombo N, Creutzberg C, and Amant F, et al (2016) ESMO-ESGO-ESTRO consensus conference on endometrial cancer: diagnosis, treatment and follow-up Ann Oncol 27(1) 16-41 https://doi.org/10.1093/annonc/mdv484

5. Gallup DG and Stock RJ (1984) Adenocarcinoma of the endometrium in women 40 years of age or younger Obstet Gynecol 64 417-421 PMID: $\underline{6462572}$

6. Crissman JD, Azoury RS, and Barnes AE, et al (1981) Endometrial cancer in women 40 years of age or younger Obstet Gynecol 57 699-704 PMID: 7015203

7. Farhi DC, Nosanchuk J, and Silverberg SG (1986) Endometrial adenocrcinoma in women under 25 years of age Obstet Gynecol 68 741-745 PMID: 3785784

8. Yamazawa K, Hirai M, and Fujito A, et al (2007) Fertility-preserving treatment with progestin, and pathological criteria to predict responses, in young women with endometrial cancer Hum Reprod 22(7) 1953-1958 https://doi.org/10.1093/humrep/dem088 PMID: 17449880

9. Navarria I, Usel M, and Rapiti E, et al (2009) Young patients with endometrial cancer: how many could be eligible for fertilitysparing treatment? Gynecol Oncol 114 448-451 https://doi.org/10.1016/j.ygyno.2009.05.038 PMID: 19560801

10. Tinelli R, Litta P, and Meir Y, et al (2014) Advantages of laparoscopy versus laparotomy in extremely obese women (BMI>35) with early stage endometrial cancer: a multicenter study Anticancer Res 34(5) 2497-2502 PMID: 24778066

11. Bogani G, Dowdy SC, and Cliby WA, et al (2016) Management of endometrial cancer: issues and controversies Eur J Gynaecol Oncol 37(1) 6-12 PMID: 27048101 
12. Walsh C (2005) Coexisting ovarian malignancy in young women with endometrial cancer Obstet Gynecol 106 693-699 https:// doi.org/10.1097/01.AOG.0000172423.64995.6f PMID: 16199623

13. Creasman WT, Morrow CP, and Bundy BN, et al (1987) Surgical pathologic spread patterns of endometrial cancer. A gynecologic oncology group study Cancer 60(8) 2035-2041 https://doi.org/10.1002/1097-0142(19901015)60:8+\&lt:2035::AIDCNCR2820601515\&gt;3.0.CO;2-8 PMID: $\underline{3652025}$

14. Chi DS, Barakat RR, and Palayekar MJ, et al (2008) The incidence of pelvic lymph node metastasis by FIGO staging for patients with adequately surgically staged endometrial adenocarcinoma of endometrioid histology Int J Gynecol Cancer 18 269-273 https://doi.org/10.1111/j.1525-1438.2007.00996.x PMID: 18334008

15. Hardesty LA, Sumkin JH, and Hakim C, et al (2001) The ability of helical CT to preoperatively stage endometrial carcinoma AJR Am J Roentgenol 176 603-606 https://doi.org/10.2214/ajr.176.3.1760603 PMID: 11222188

16. DelMaschio A, Vanzulli A, and Sironi S, et al (1993) Estimating the depth of myometrial involvement by endometrial carcinoma: efficacy of tranvaginal sonography vs MR imaging AJR Am J Roentgenol 160 533-538 https://doi.org/10.2214/ajr.160.3.8430547 PMID: 8430547

17. Yamashita $\mathrm{Y}$, Mizutani $\mathrm{H}$, and Torashima M, et al (1993) Assessment of myometrial invasion by endometrial carcinoma: tranvaginal sonography vs. Contrast-enhanced MR imaging AJR Am J Roentgeno/ 161 595-599 https://doi.org/10.2214/ajr.161.3.8352114 PMID: 8352114

18. Saez F, Urresola A, and Larena JA, et al (2000) Endometrial carcinoma: assessment of myometrial invasionwith plain and gadolinum-enhanced MR imaging J Magn Reson Imaging 12 460-466 https://doi.org/10.1002/1522-2586(200009)12:3\&lt:460::AIDJMRI12\&gt;3.0.CO;2-3 PMID: 10992314

19. Zarbo G, Caruso G, and Caruso S, et al (2000) Endometrial Cancer: prospective evaluation of myometrial infiltration: magnetic resonance imaging versus tranvaginal sonography Eur J Gynaecol Oncol 21 95-97

20. Alcázar JL, Gastón B, and Navarro B, et al (2017) Transvaginal ultrasound versus magnetic resonance imaging for preoperative assessment of myometrial infiltration in patients with endometrial cancer: a systematic review and meta-analysis $\mathrm{J}$ Gynecol Oncol 28(6) e86 https://doi.org/10.3802/jgo.2017.28.e86 PMID: 29027404 PMCID: $\underline{5641536}$

21. Kaku T, Yoshikawa $\mathrm{H}$, and Tsuda $\mathrm{H}$, et al (2001) Conservative therapy for adenocarcinoma and atypical endometrial hyperplasia of the endometrium in young women: central pathologic review and treatment outcome Cancer Lett 167 39-48 https://doi. org/10.1016/S0304-3835(01)00462-1 PMID: 11323097

22. Kullander S (1992) Treatment of endometrial cancer with GnRH analogues Recent Results Cancer Res 124 69-73 https://doi. org/10.1007/978-88-470-2186-0_ 7

23. Emons G, Schröder B, and Ortmann O, et al (1993) High affinity binding and direct antiproliferative effects of luteinizing hormone-releasing hormone analogs in human endometrial cancer cell lines J Clin Endocrinol Metab 77 1458-1464 PMID: 8263128

24. Jeong JW (2013) Steroid hormone intervenes in the endometrial tumorigenesis of pten ablation J Cancer Prev 18(4) 313-321 https://doi.org/10.15430/JCP.2013.18.4.313

25. De Agustín LC, Sastre FL, Galán VC, et al (2008) Conservative management of patients with early endometrial carcinoma: a systematic review Clin Trans/ Oncol 10 155-162 https://doi.org/10.1007/s12094-008-0173-1

26. Podratz KC, O'Brien PC, and Malkasian GD Jr, et al (1985) Effects of progestational agents in treatment of endometrial carcinoma Obstet Gynecol 66 106-110 PMID: 4011061

27. Saegusa M and Okayasu I (1998) Progesterone therapy for endometrial carcinoma reduces cell proliferation but does not alter apoptosis Cancer 83 111-121 https://doi.org/10.1002/(SICI)1097-0142(19980701)83:1\&lt:111::AID-CNCR15\&gt:3.0.CO;2-\# PMID: 9655300 
28. Florio P, Imperatore A, and Litta P, et al (2010) The use of nomegestrol acetate in rapid preparation of endometrium before operative hysteroscopy in pre-menopausal women Steroids 75(12) 912-917 https://doi.org/10.1016/j.steroids.2010.05.009 PMID: 20566359

29. Vereide AB, Kaino T, and Sager G, et al (2005) Scottish gynaecological clinical trials group. Bcl-2, BAX, and apoptosis in endometrial hyperplasia after high dose gestagen therapy: a comparison of responses in patients treated with intrauterine levonorgestrel and systemic medroxyprogesterone Gynecol Oncol 97(3) 740-750 https://doi.org/10.1016/j.ygyno.2005.02.030 PMID: 15885761

30. Ferrandina G, Zannoni GF, and Gallotta V, et al (2005) G.Progression of conservatively treated endometrial carcinoma after full term pregnancy: a case report Gynecol Oncol 99(1) 215-217 https://doi.org/10.1016/j.ygyno.2005.05.025 PMID: 15979130

31. Kimmig R, Strowitzki T, and Muller-Hocker J, et al (1995) Conservative treatment of endometrial cancer permitting subsequent triplet pregnancy Gynecol Oncol 58 255-257 https://doi.org/10.1006/gyno.1995.1221 PMID:

32. Randall TC and Kurman RJ (1997) Progestin treatment of atypical hyperplasiaand well-differentiated carcinoma of the endometrium in women under age 40 Obstet Gynecol $90434-440$ https://doi.org/10.1016/S0029-7844(97)00297-4 PMID: 9277658

33. Ramirez PT, Frumovitz M, and Bodurka DC, et al (2004) Hormonal therapy for the management of grade 1 endometrial adenocarcinoma: a literature review Gynecol Oncol 95 133-138 https://doi.org/10.1016/j.ygyno.2004.06.045 PMID: 15385122

34. Mao Y, Wan X, and Chen $\mathrm{Y}$, et al (2010) Outcomes of conservative therapy for young women with early endometrial adenocarcinoma Fertil Steril 93(1) 283-285 https://doi.org/10.1016/j.fertnstert.2009.07.999

35. Jadoul $P$ and Donnez J (2003) Conservative treatment may be beneficial for young women with atypical endometrial hyperplasia or endometrial adenocarcinoma Fertil Steril 80 1315-1324 https://doi.org/10.1016/S0015-0282(03)01183-X PMID: 14667859

36. Montz FJ, Bristow RE, and Bovicelli A, et al (2002) Intrauterine progesterone treatment of early endometrial cancer Am J Obstet Gynecol 186 651-657 https://doi.org/10.1067/mob.2002.122130 PMID: $\underline{11967486}$

37. Šparac V, Ujevi B, and Ujevi M, et al (2006) Successful pregnancy after hysteroscopic removal of grade I endometrial carcinoma in a young woman with Lynch syndrome Int J Gynecol Cancer 16(1) 442-445 https://doi.org/10.1111/j.1525-1438.2006.00219.x PMID: 16515642

38. Vilos GA, Ettler HC, and Edris F, et al (2007) Endometrioid adenocarcinoma treated by hysteroscopic endomyometrial resection J Minim Invasive Gynecol 14(1) 119-122 https://doi.org/10.1016/j.jmig.2006.09.004 PMID: 17218243

39. Mazzon I, Corrado G, and Masciullo V, et al (2010) Conservative surgical management of stage IA endometrial carcinoma for fertility preservation Fertil Steril 93 1286-1289 https://doi.org/10.1016/j.fertnstert.2008.12.009

40. Jauniaux E, Collins S, and Burton GJ (2018) Placenta accreta spectrum: pathophysiology and evidence-based anatomy for prenatal ultrasound imaging Am J Obstet Gynecol 218(1) 75-87 https://doi.org/10.1016/j.ajog.2017.05.067

41. Alonso S, Castellanos T, and Lapuente F, et al (2015) Hysteroscopic surgery for conservative management in endometrial cancer: a review of the literature Ecancermedicalscience 9505 https://doi.org/10.3332/ecancer.2015.505 PMID: 25729418 PMCID: 4335962

42. Vandenput I, Van Eygen K, and Moerman P, et al (2009) Ineffective attempt to preserve fertility with a levonorgestrel-releasing intrauterine device in a young woman with endometrioid endometrial carcinoma: a case report and review of the literature Eur J Gynaecol Oncol 30(3) 313-316 PMID: 19697629

43. Tanmahasamut $P$ and Wongwananuruk $T$ (2010) Challenging regimen for long-term conservative treatment of endometrial adenocarcinoma in young women: a case report and review of the literature Case Rep Oncol 3(3) 380-385 https://doi. org/10.1159/000321731 PMID: $\underline{21113347}$ PMCID: $\underline{2992425}$ 
44. Minig L, Franchi D, and Boveri S, et al (2011) Progestin intrauterine device and GnRH analogue for uterus-sparing treatment of endometrial precancers and well-differentiated early endometrial carcinoma in young women Ann Oncol 22(3) 643-649 https:// doi.org/10.1093/annonc/mdq463

45. Obermair et al Improving the treatment for women with early stage cancer of the uterus (feMMe) [http://ClinicalTrials. gov"ClinicalTrials.gov] Identifying number: NCT 01686126

46. Imai M, Jobo T, and Sato R, et al (2001) Medroxyprogesterone acetate therapy for patients with adenocarcinoma of the endometrium who wish to preserve the uterus-usefulness and limitations Eur J Gynaecol Oncol 22 217-220

47. Wang CB, Wang CJ, and Huang HJ, et al (2002) Fertility-preserving treatment in young patients with endometrial adenocarcinoma Cancer 94 2192-2198 https://doi.org/10.1002/cncr.10435 PMID: 12001117

48. Benshushan A, Paltiel O, and Brzezinski A, et al (2001) Ovulation induction and risk of endometrial cancer: a pilot study Eur J Obstet Gynecol Reprod Biol 9 53-57 https://doi.org/10.1016/S0301-2115(01)00344-X

49. Lowe MP, Cooper BC, and Sood AK, et al (2003) Implementation of assisted reproductive technologies following conservative management of FIGO grade I endometrial adenocarcinoma and/or complex hyperplasia with atypia Gynecol Oncol 91(3) 569-572 https://doi.org/10.1016/j.ygyno.2003.08.007 PMID: 14675678 